\title{
Nasal Cavity Olfactory Neuroblastoma
}

National Cancer Institute

\section{Source}

National Cancer Institute. Nasal Cavity Olfactory Neuroblastoma. NCI Thesaurus. Code C7604.

An olfactory neuroblastoma arising in the nasal cavity. 